\title{
Impact of the Drag Force and the Magnus Effect on the Trajectory of a Baseball
}

\author{
Haiduke Sarafian \\ The Pennsylvania State University, University College, York, PA, USA \\ Email: has2@psu.edu
}

Received 10 March 2015; accepted 10 April 2015; published 14 April 2015

Copyright (C 2015 by author and Scientific Research Publishing Inc.

This work is licensed under the Creative Commons Attribution International License (CC BY). http://creativecommons.org/licenses/by/4.0/

c) (i) Open Access

\begin{abstract}
We consider the impact of drag force and the Magnus effect on the motion of a baseball. Quantitatively we show how the speed-dependent drag coefficient alters the trajectory of the ball. For the Magnus effect we envision a scenario where the rotation of the ball confines the Magnus force to the vertical plane; gravity, drag force and the Magnus force make a trio-planar system. We investigate the interplay of these forces on the trajectories.
\end{abstract}

\section{Keywords}

\section{Drag Force, Magnus Effect, Spinning Ball, Baseball, Nonlinear Physics, Mathematica}

\section{Motivation and Introduction}

In introductory physics, engineering and math courses, undergraduate students are traditionally introduced to the concept of projectile motion such that a projectile is thrown at an angle in a vertical plane in a vacuum. The motion in the vacuum is analyzed because in the absence of air, utilizing the Newton's second law yields to a set of two independent second-order linear ODEs. The trivial solution of these equations provides information about the kinematics of the projectile, such as the trajectory, range, time of flight, etc. [1]. If one applies the learned concepts to a real-life situation such as a pitched and/or a batted baseball, one disappointingly would be surprised realizing the gross discrepancies of the two scenarios. For instance, a $45^{\circ}$ batted baseball does not maximize the range, and the trajectory of a baseball is not a symmetric parabola.

The motivation of this article stems from the former question, namely "At what angle above the horizontal a baseball should be batted making the range a maximum?" To answer this question, we analyze the problem systematically. In the course of our analysis, we stumbled upon a few interesting related issues. To the author's amazement, the analysis led also to uncharted territories unveiling features of a flying baseball that to date have not been reported in scientific literature. In this article we confirm the previously reported quantitative results 
and extend the investigation for the "what-if" scenarios.

\section{Physics of a Projectile in the Air; The Impact of Air Resistance and the Spin}

We consider a ball projected in the air at an arbitrary initial angle above the horizontal. In addition to gravity the ball encounters air resistance. Irrespective of its speed, the drag force (the air resistance) acts in the opposite direction of the motion retarding its movement. In Figure 1, a snapshot of a flying ball with its instantaneous velocity and the drag force are shown with $\boldsymbol{v}$ and $\boldsymbol{F}_{D}$, respectively. In practice a batted ball also spins; it may spin backward (backspin) or forwards (topspin). We quantify the spin by its angular velocity $\boldsymbol{\omega}$. We consider a spinning ball with angular velocity vector perpendicular to the vertical plane. Hence, a back-spin ball orients its $\boldsymbol{\omega}$ parallel to the ground and outward to the vertical plane. Conversely, a top-spun ball orients its $\omega$ parallel and inward to the ground. Figure 1 displays a back-spinning (counter-clockwise rotating) ball. The dot at the center of the ball represents the head of the $\omega$ arrow emerging the $x z$-plane. For the chosen scenario, the spinning ball is subject to an additional force; a spin-dependent force, namely the Magnus force [2]. This force is in proportion to $\boldsymbol{F}_{M} \sim \boldsymbol{\omega} \times \boldsymbol{v}$. Accordingly, this force also lies in the vertical plane making the three active forces namely, $\boldsymbol{w}$, $\boldsymbol{F}_{D}$ and $\boldsymbol{F}_{M}$ a trio coplanar. For the given scenario the ball stays in the vertical plane, and therefore, the analysis of the problem becomes two-dimensional.

Figure 1 visually assists distinguishing the impact of each individual force. For instance, it shows the horizontal components of the drag and the Magnus force collectively are acting along the opposite direction of the motion; they have a retarding impact. While the vertical component of the drag force makes the baseball "heavier", the vertical component of the Magnus force acting along the upward direction acts as it makes the baseball "lighter". It is this interplay of the components of the forces that makes the analysis of the problem challenging. The relative quantitative strengths of these components depend on the parameters describing the flying ball and the media. In Section 3 for a chosen set of parameters we address these issues. It needs to be mentioned that for a baseball thrown in a vacuum there are no such challenges; the only active force is gravity!

Here we consider the characteristics of the last two forces. For speeds relevant to the MLB (Main League

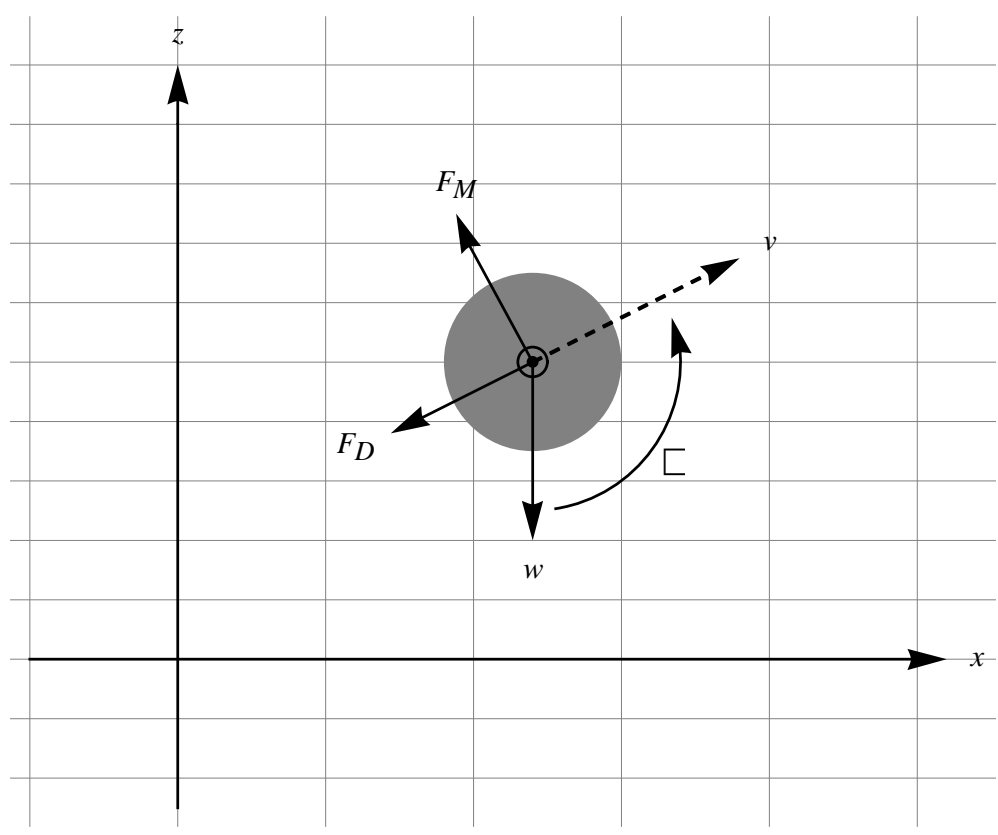

Figure 1. The profile of a flying baseball. Its back-spin (counter-clockwise rotation) is shown with a vector piercing outward and perpendicular to the vertical $x z$-plane. The head of the angular velocity vector $\omega$ is shown with a black dot at the center of the ball circumvented with a gray circle. The dashed line is the instantaneous velocity of the baseball; the solid arrows are the active forces. From the top in the counter-clockwise direction the lines are: the Magnus force $\boldsymbol{F}_{M}$, the Drag force $\boldsymbol{F}_{D}$ and the weight $W$. 
Baseball) the airflow around a baseball is considered as laminar [3]; therefore, the drag force is formatted according to $\boldsymbol{F}_{D}=\frac{1}{2} C_{D} \rho A v(-v)$. This equation explicitly shows the drag force at any instance is along the opposite direction of motion. The parameters in this equation are $C_{D}, \rho, A$, the drag coefficient, the density of the media (air) and the cross sectional area of the ball i.e. $\pi R^{2}$, where $R$ is the radius of the ball. Utilizing a Computer Algebra System (CAS) recently this equation has been applied to a "real-life" situation. However, assuming a speed independent coefficient the impact of the drag force has been trivialized leading to inaccurate conclusions [4]. On the other hand, considering speed dependent real-life data, the drag coefficient and therefore the drag force is parameterized leading to a good phenomenological approximation [5]; in our analysis we utilize the latter. In the course of our search we encounter $\frac{1}{m} F_{D}$ where $\mathrm{m}$ is the mass of the baseball. According to [5], this ratio is equated to $\frac{1}{m} F_{D}=f(v) v^{2}$, where $f(v) \equiv \frac{1}{2 m} C_{D} \rho A=0.0039+\frac{0.0058}{1+\mathrm{e}^{\frac{v-v_{d}}{\Delta}}}$ with $v_{d}=35$ and $\Delta=5$ both in $\mathrm{m} / \mathrm{s}$ units. Noting in SI, $f(v)$ has the units of $[f(v)]_{\mathrm{SI}}=$ met $^{-1}$. The author being a nuclear physicist realizes that aside from an overall sign this function is somewhat similar to the potential describing certain nuclear reactions [6]. Given this parametrization we solve $f(v)$ for $C_{D}$; this yields $C_{D}=(2 m) /(r A) f(v)$. Noting, for slow speeds, $C_{D}$ approaches $\sim 0.52$ i.e. $\operatorname{Limit}_{v \rightarrow \text { mall }} C_{D}^{\mathrm{TM}} 0.52$. Utilizing the density of the air and the radius of a baseball in SI units, namely $\{\rho, R\}=\left\{1.23 \mathrm{~kg} / \mathrm{m}^{3}, 3.64 \times 10^{-2} \mathrm{~m}\right\}$ Figure 2 displays how $C_{D}$ varies vs. the practical range of speed.

According to Figure 2, there are three ranges of interest: slow, medium and fast. These correspond to the speeds: $10<v(\mathrm{~m} / \mathrm{s})<20,20<v(\mathrm{~m} / \mathrm{s})<50$ and $v(\mathrm{~m} / \mathrm{s})>50$, respectively. The corresponding $C_{D}$ 's for the first and the last range are constants and are as high as 0.52 and as low as 0.22 , respectively. The middle range is the transition range where the $C_{D}$ varies from the high value of 0.52 to the low value of 0.22 . Consequently, a baseball batted at a typical MLB speed $\sim 88 \mathrm{MPH}(=37 \mathrm{~m} / \mathrm{s})$ encounters a weak drag force at the beginning, and while traversing along its trajectory continuously slows down encountering a relatively strong drag force. In short, the impact of the drag force is not constant. One intuitively expects the impact of the drag force at the beginning to be less than at the end of the flight. In Section 3, quantitatively we validate our intuition.

The other force is the Magnus force, for a baseball it is parametrized as $\boldsymbol{F}_{M}=S \boldsymbol{\omega} \times \boldsymbol{v}$, where $S$ is independent of speed and is a constant [5]. The $S$ has a dimension of mass and in SI units is $[S]_{\mathrm{SI}}=\mathrm{kg}$. It is interesting to note in most literature this force is formulated as $F_{M} \sim \omega v^{2}$. However, the latter format simply reduces to the former. To see the equivalence of these two formats one may think that in a resting state of the baseball where

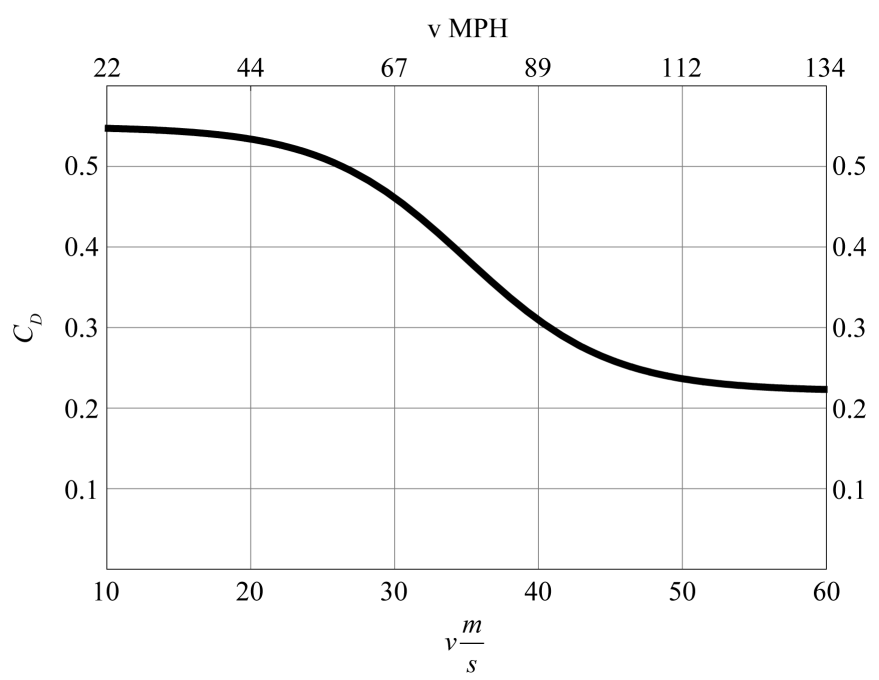

Figure 2. Variation of the drag coefficient for a baseball vs. speed. The lower horizontal axis is in $\mathrm{m} / \mathrm{s}$; the upper horizontal axis is the corresponding speed in MPH. 
the ball is standing still and the air passes it by with the velocity $v$ from right to left; see Figure 1 . A point at the top edge of the ball moves with a fast speed, $v+R \omega$, while its counterpoint at the bottom of the baseball moves with a slower speed, $v-R \omega$. The difference between these two speeds according to $v^{2}$ format yields $\sim(v+R \omega)^{2}-(v-R \omega)^{2} \sim R \omega v$. Therefore, the $S$ is the lump sum of the relevant factors: the lift coefficient, the cross section area of the baseball and the density of the air yielding the former format, namely $F_{M}=S \omega v$. To determine the orientation of the Magnus force one also may utilize Bernoulli's fluid mechanics law [1]. Paraphrasing, the law states "a fast moving fluid corresponds to a low pressure and vice versa". Since the top points on a ball move at a higher speed vs. the bottom points, the pressure difference would have a tendency to push the ball upward. Consequently, the corresponding force for a back-spinning baseball is $\boldsymbol{F}_{D}=S \boldsymbol{\omega} \times \boldsymbol{v}$; its direction is depicted in Figure 1. In practice, similar to the drag force we encounter the value of $\frac{1}{m} F_{M}$; this is written as $\frac{1}{m} \boldsymbol{F}_{M}=B \boldsymbol{\omega} \times \boldsymbol{v}$ where phenomenological unit-less value $B$ for a baseball of mass $m=143 \mathrm{~g}$ is $B=4.1 \times 10^{-4}$ [5]. The other relevant factor to the Magnus force is the spin value, $\omega$. It is noted [7] that a pitched baseball on its way to a batter spins once per five feet. Knowing the distance between the pitcher's mound, home base and a typical flight time yield to an accepted value $\omega=1800$ RPM. Furthermore, it is also assumed a pitched or a batted baseball on its trajectory sustains its spin. In other words the value of $\omega$ is constant. With this detailed phenomenological information about the parameters describing the drag and the Magnus force in the next section we cast the equations describing the motion of the baseball.

\section{Formulation of the Physics of a Batted Baseball}

As we explained in the previous section a batted back-spun baseball with its initial angular velocity vector parallel to the horizontal flies and stays in a vertical plane. A vertical plane is the one that contains the initial velocity vector. In other words, the motion of the ball occurs in a 2D space. One such plane with a Cartesian $x z$ coordinate is shown in Figure 1. We utilize the active forces depicted in Figure 1 and compose the equations conducive to the analysis of the motion of the batted ball. Utilizing Newton's second law we write $\boldsymbol{F}_{\text {net }}=m \boldsymbol{a}$. This equation explicitly reads, $m \ddot{\boldsymbol{r}}=\boldsymbol{W}+\boldsymbol{F}_{D}+\boldsymbol{F}_{M}$, where the over double-dots means the second order time derivative. We substitute the forces from the previous section yielding $m \ddot{\boldsymbol{r}}=m \boldsymbol{g}+m f(v) v(-\boldsymbol{v})+S \boldsymbol{\omega} \times \boldsymbol{v}$. Since the velocity stays always in the vertical plane we write $v=\{\dot{x}, 0, \dot{z}\}$. On the other hand, as we discussed the angular velocity sustains its character, meaning, its value and its orientation stay the same. In a right handed coordinate system shown in Figure 1, we write $\omega=\{0,-\omega, 0\}$. This yields,

$\boldsymbol{\omega} \times \boldsymbol{v}=\{0,-\omega, 0\} \times\{\dot{x}, 0, \dot{z}\}=\{-\omega \dot{z}, 0, \omega \dot{x}\}$. The $x$ and the $z$ components of the force equation yields,

$$
\begin{gathered}
\ddot{x}=-f(v) v \dot{x}-B \omega \dot{z} \\
\ddot{z}=-g-f(v) v \dot{z}+B \omega \dot{x},
\end{gathered}
$$

Note the last term of Equations (1), (2) are only operative for a spinning baseball. In other words, the equations associated with a non-spinning baseball are simpler than those given by Equations (1), (2). For a back-spun ball the horizontal components of the drag and the Magnus force are constructively additive, while along the vertical direction they are destructive. Equations (1), (2) describe the motion of the baseball. These are highly coupled non-linear ODEs. The solution of these equations are conducive to a set of explicit time dependent coordinates, namely $\{x(t), z(t)\}$. To highlight the degree of difficulty and the challenge of the task at head, we write these equations explicitly.

$$
\begin{gathered}
\ddot{x}=-\left(\alpha+\frac{\beta}{1+\mathrm{e}^{\frac{1}{\Delta}\left(\sqrt{\dot{x}^{2}+\dot{z}^{2}}-v_{d}\right)}}\right) \sqrt{\dot{x}^{2}+\dot{z}^{2}} \dot{x}-B \omega \dot{z} \\
\ddot{z}=-g-\left(\alpha+\frac{\beta}{1+\mathrm{e}^{\frac{1}{\Delta}\left(\sqrt{\dot{x}^{2}+\dot{z}^{2}}-v_{d}\right)}}\right) \sqrt{\dot{x}^{2}+\dot{z}^{2}} \dot{z}+B \omega \dot{x}
\end{gathered}
$$

where $\alpha$ and $\beta$ have dimensions of $\mathrm{L}^{-1}$ while $v_{d}$ and $\Delta$ have dimensions $\mathrm{L} \mathrm{T}^{-1}$; all four parameters are 
constant. It is obvious that a set of such equations may not have symbolic solutions! Despite of our insistence we were unable to convert these equations to solvable entities. We then apply Mathematica symbolic DSolve command, but it also fails to produce analytic solutions. As a last resource we attempt solving them numerically. We introduce a set of meaningful, practical initial conditions and utilize Mathematica's numeric routine, namely, NDSolve. This simple seven letter word successfully produces an output. Utilizing Figure 2, we consider a typical initial speed of $88 \mathrm{MPH}$ which corresponds to $37 \mathrm{~m} / \mathrm{s}$. Our first objective is to evaluate the initial batted angle that maximizes the range; therefore, in our calculation we treat the initial angle as a variable. Also, we insert two switches in the code. By turning them on and off we include or exclude the drag and the Magnus forces one at a time. The switches are listed as $\{\alpha D, \beta M\}$. This ordered list with possible variations are: $\{0,0\},\{0,1\}$, $\{1,0\}$ and $\{1,1\}$. $\{$ drag $=0$, Magnus $=0\}$ represents the vacuum; $\{$ drag $=0$, Magnus $\neq 0\}$ represents the impact of the pure Magnus effect; $\{d r a g \neq 0$, Magnus $=0\}$ represents the impact of the pure drag force; and $\{d r a g \neq 0$, Magnus $\neq 0\}$ represents the impact of the combined drag and the Magnus forces, respectively. The relevant parameters in SI units are:

$$
\begin{gathered}
\text { values }=\left\{\alpha \rightarrow 0.0039, \beta \rightarrow 0.0058, v_{d} \rightarrow 35, \Delta \rightarrow 5, \rho \rightarrow 1.23, R \rightarrow 36.410^{-3},\right. \\
\left.m \rightarrow 0.145, g \rightarrow 9.8, B b \rightarrow 4 \times 110^{-4}, \omega \rightarrow 1800 \cdot \frac{\pi}{30}, v_{0} \rightarrow 37\right\}
\end{gathered}
$$

The initial condition is comprised of the specifications of the initial velocity; on the vertical $x z$-plane this is $\boldsymbol{v}_{0}=\left\{v_{0} \operatorname{Cos}[\theta], 0, v_{0} \operatorname{Sin}[\theta]\right\}$. For four possible switch settings and for each of the chosen initial angles within the range of $\{10,20,30,40,50,60,70,80,90\}^{\circ}$ we solve the corresponding equations. Utilizing these solutions we also deduce the time of flight.

The initial angles are discrete, i.e. they are $10^{\circ}$ apart and so are their corresponding ranges. Utilizing Interpolation command we fill in the gap and generate the needed continuous coordinates. With these continuous coordinates on hand we display the discrete solutions along with the interpolated continuous spectrum. These are shown in Figure 3.

With these plots it is easy to draw a few conclusions. For instance, the first and the last plots depict the two extreme scenarios. The first graph corresponds to the vacuum, and the last graph shows the impact of the combined drag and the Magnus effects. The difference between these two scenarios is quite pronounced. Their maximum range not only occurs at two different angles, but their numeric values are almost off by a factor of two. Similarly, one may also compare the second dot to the third plot. Both figures describe the impact of the media; the second plot is with the pure Magnus effect, while the third plot shows the impact of the pure drag force. Although these scenarios do not represent real-life situations they illustrate the impact of the individual forces.

Furthermore, with the interpolated continuous function at hand we search for the initial angle that maximizes the range. The output of the search is tabulated in Table 1.

The first column of Table 1 shows the maximum range occurs at $45^{\circ}$; this is a "classics" knowledge and is an indicative of the accuracy of our analysis. For a real-life scenario, the fourth column shows quite a different result. For a projectile batted at $37 \mathrm{~m} / \mathrm{s}$ with a 1800 RPM the angle that maximizes the range is $35^{\circ}$ i.e. it is less by $10^{\circ}$ vs. the classic value. The numeric entries in the second and third columns are helpful to draw conclusions about the impact of the individual active forces.

Utilizing the corresponding time of fight in Figure 4 we display time of flights vs. the departure angles.

These figures are self-explanatory. The headings are the same as in Figure 3. These plots clearly show the impact of the forces on the time of flight.

Finally, utilizing the solutions of Equations (1), (2), namely $\{x(t), y(t)\}$ we apply ParametricPlot command and plot the trajectories of the balls. These are shown in Figure 5.

Table 1. The first row is the maximum range in meters; the second row shows the corresponding initial angle in degrees. The heading of the matrix explicitly describes the scenario of interest.

\begin{tabular}{ccccc}
\hline & $\{\alpha D, \beta M\}=00$ & $\{\alpha D, \beta M\}=01$ & $\{\alpha D, \beta M\}=10$ & $\{\alpha D, \beta M\}=11$ \\
\hline “max range, m-->” & 139.694 & 162.579 & 78.2832 & 84.095 \\
$\theta^{\circ}$ “-->” & $\theta \rightarrow 45$ & $\theta \rightarrow 38.3973$ & $\theta \rightarrow 39.4280$ & $\theta \rightarrow 35.1726$
\end{tabular}




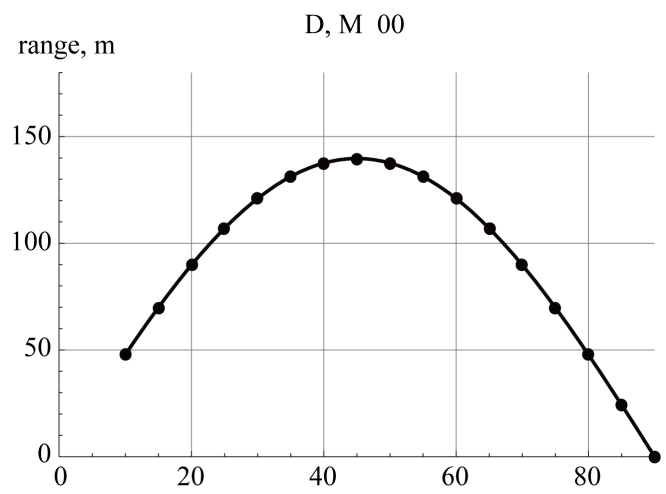

D, M 10

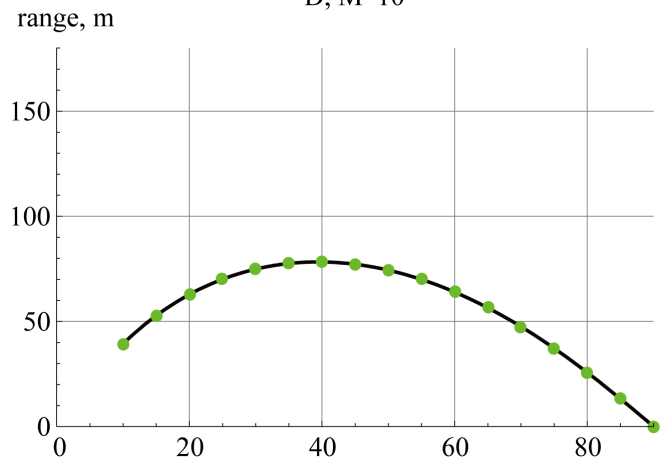

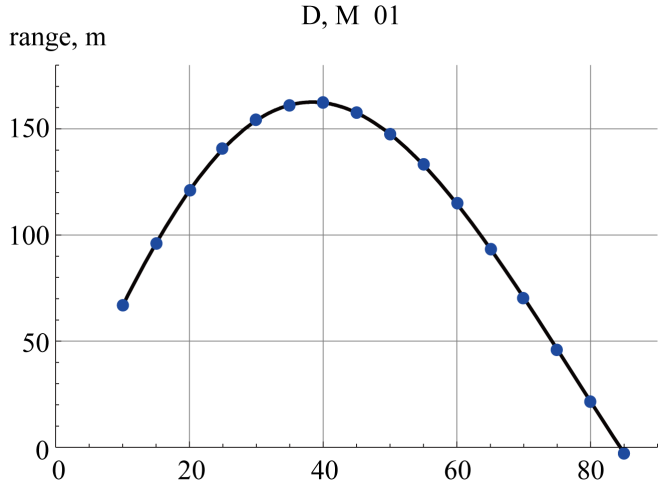

D, M 11

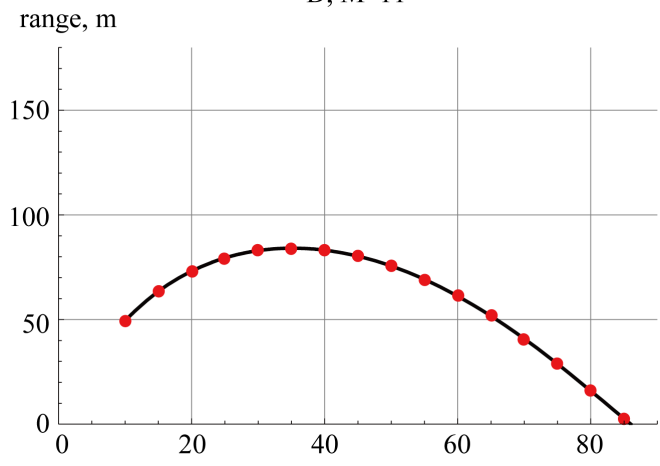

Figure 3. The dots are the numeric solutions of Equations (1), (2) corresponding to the switch setting $\{\alpha D, \beta M\}$. The solid curve in each plot is the corresponding interpolated functions.

time of flight, $\mathrm{S} \quad \mathrm{D}, \mathrm{M} 00$

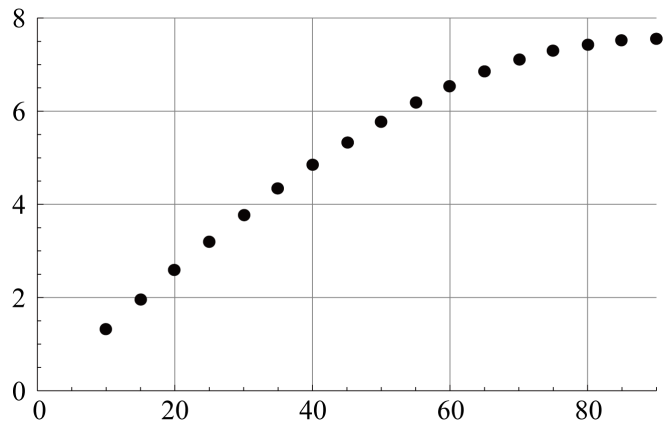

D, M 10

time of flight, $\mathrm{s}$

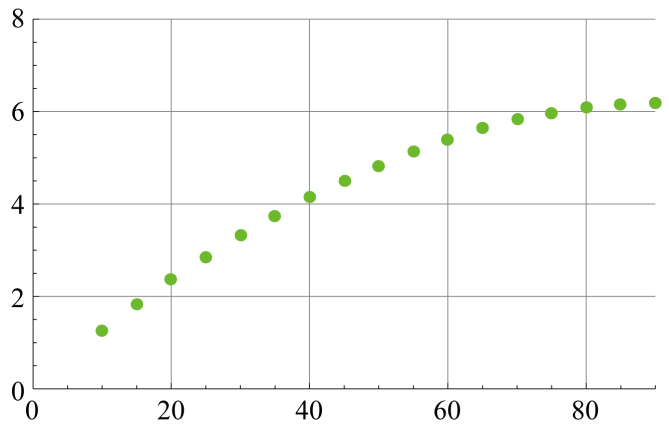

D, M 01

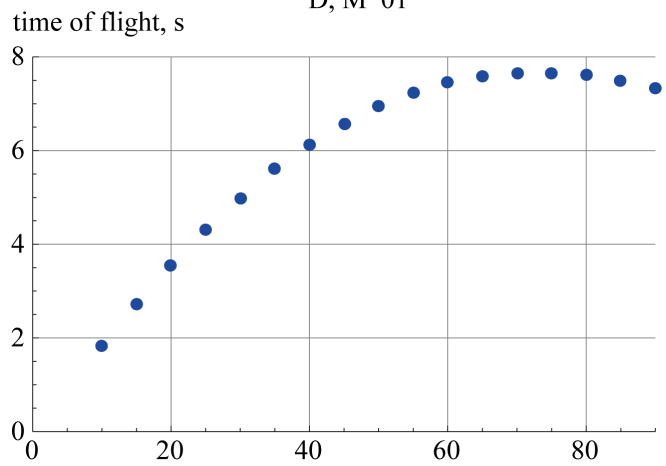

D, M 11

time of flight, $\mathrm{s}$

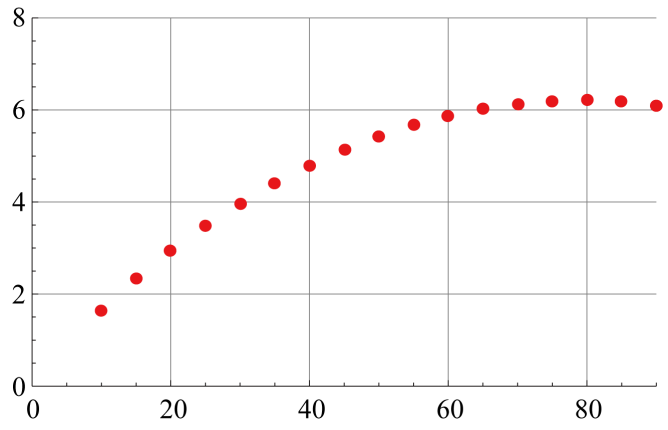

Figure 4. Plots of time of flights vs. the initial angles for four cases of interest. 

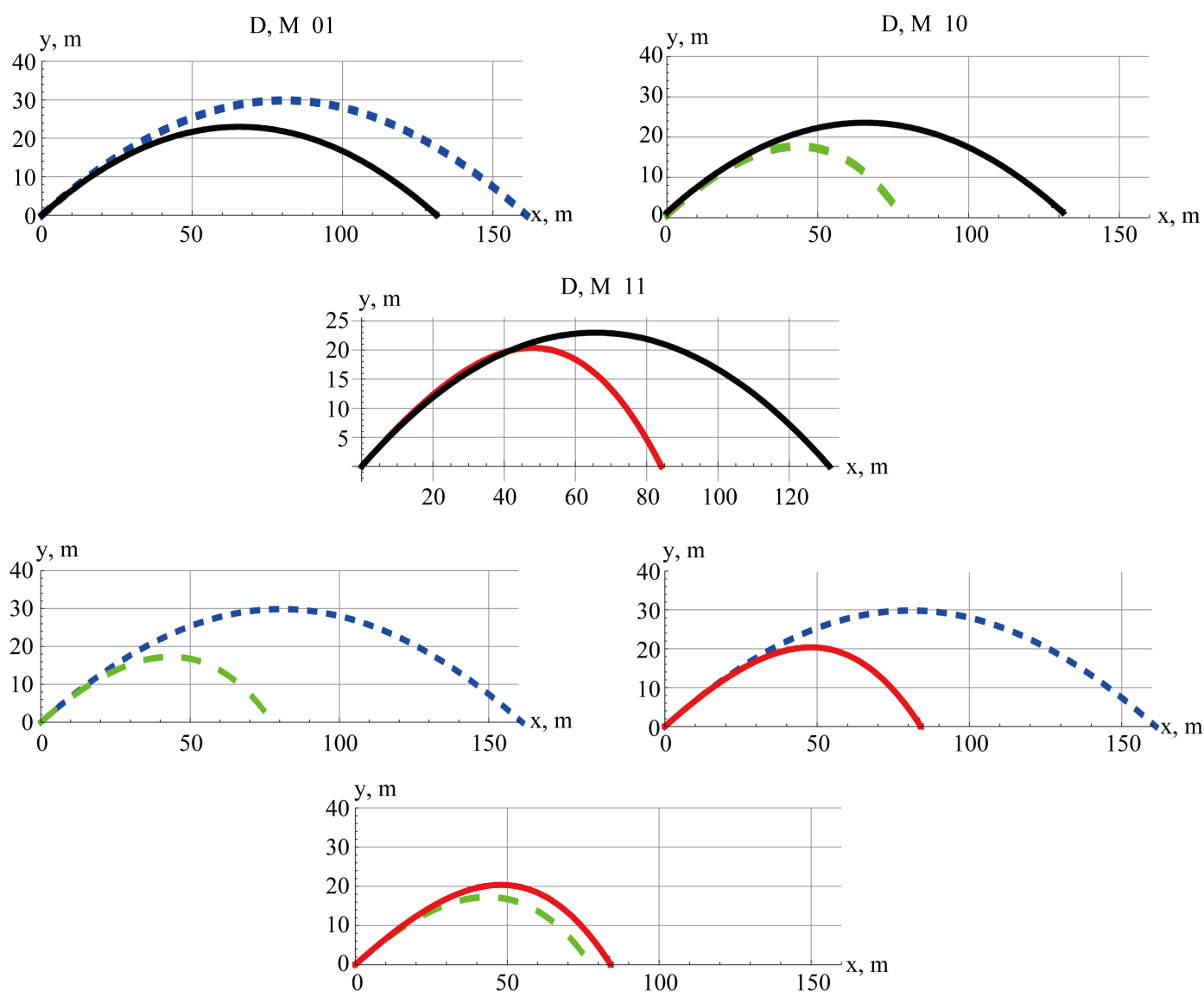

Figure 5. The black curve is the trajectory in a vacuum and is used as a reference. The color code is the same as Figure 3.

Trajectories depicted in Figure 5 are associated with the initial projectile angle $35^{\circ}$. These graphs show the impact of the various scenarios on the trajectory. For instance, the red trajectory is being compared to the black trajectory. The former shows the impact of the combined drag and the Magnus force, while the latter is the trajectory in a vacuum. These two trajectories are indistinguishable for the greater part of the first half of their pathway and deviate more in the second half. After deviating paths, the red curve bends severely, while the black one follows its "natural" curvature. The reasoning for these behaviors are tied to the speed dependent drag coefficient depicted in Figure 2. Simply put, initially, a batted baseball flies quickly, and because of the small value of the drag coefficient it encounters a small drag force. Conversely, while it traverses along its trajectory, the loss of its speed is associated with a large drag coefficient and hence it encounters a stronger drag force. The latter is the main cause of the deviation from the counterpart in a vacuum. For the rest of the graphs depicted in Figure 5 one may easily draw the appropriate conclusions. The author believes these need to be done based on the reader's interest.

We evaluate the maximum height, the results are shown pictorially in Figure 6.

Figure 6 contains interesting information. For instance, the blue labels for any chosen initial angle are higher than the rest of the colored labels; this is because the former excludes the drag force. These are also higher than the black labels showing the lifting impact of the Magnus force. The green and the red labels are quite lower than the black ones; this is because the former set contains the drag force and the latter is projected in a vacuum. Also we note the drastic difference between the black and the red labels. That is to say there are huge differences between the vacuum and a real-life scenario. We also note as one should expect intuitively the absolute maximum heights irrespective of the chosen scenario occurs at $90^{\circ}$, i.e. an upright projection. A further detailed study of the features displayed in Figure 6 may also reveal additional detailed information; however, the author leaves them for the interested reader to explore. 


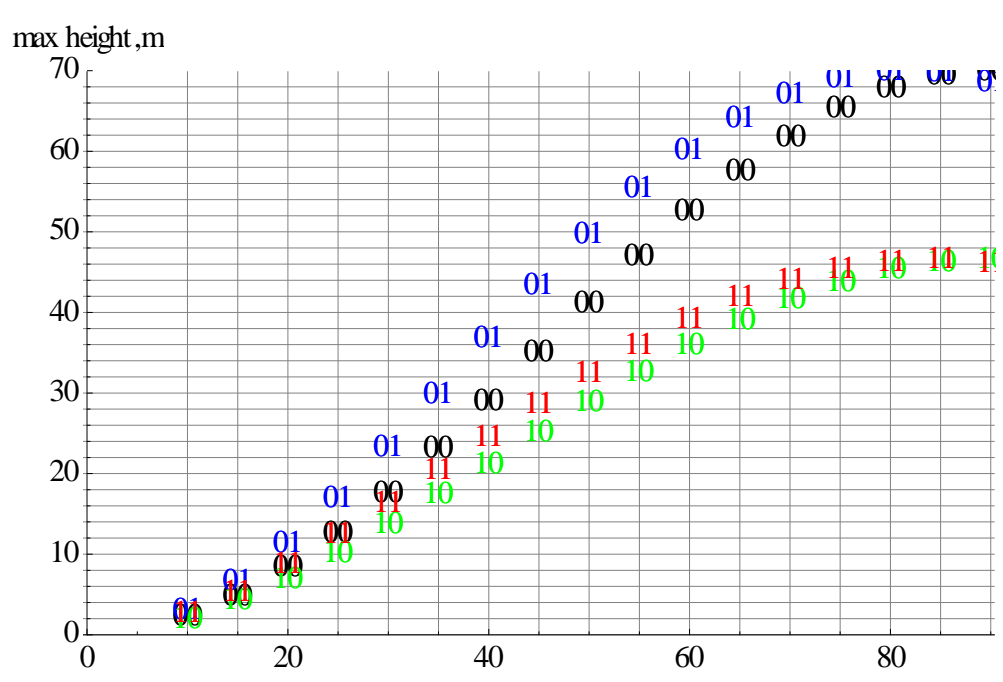

Figure 6. A consolidated plot of the maximum heights vs. the projected angles. The color codes are the same as Figure 3.

We continue exploring some more properties of a batted baseball, namely the arc length of a projectile. The length of the projectile comes about from the integration of the arc length element along the trajectory; $\mathrm{d} \ell=\sqrt{\mathrm{d} x^{2}+\mathrm{dz}^{2}}$. However, the coordinates of the projectile are implicit time-dependent functions i.e. $\{x(t), z(t)\}$ and hence we write $\mathrm{d} \ell=\sqrt{\dot{x}(t)^{2}+\dot{z}(t)^{2}} \mathrm{~d} t$, this yields $\ell=\int_{t_{1}}^{t_{2}} \sqrt{\dot{x}(t)^{2}+\dot{z}(t)^{2}} \mathrm{~d} t$. We set $\left\{t_{1}, t_{2}\right\} \equiv$ \{0, flighttime\} yielding the length. Results are shown in Figure 7.

Having these plots on hand, one may now ask at least two questions. The first questions is "At what angle a ball should be batted to gain the maximum arc length?”. The results are tabulated in Table 2.

According to Table 2 the longest trajectory arc length is associated with the second scenario. This makes intuitive sense because in the absence of the drag force the Magnus effect partially "lifts" the ball making it travel longer causing it to gain the longest arc length. Similar self-explanatory information may be deduced for other cases as well. It is reassuring to note that the value of the angle and its associated arc length in a vacuum are exactly what the author reported previously [8].

The second question stems from a careful analysis of the last plot of Figure 7. This graph shows that there are a certain set of initial projectile angles corresponding to the same arc lengths. To make the point, in Figure 8 we have drawn three horizontal lines. Each of these lines intersects the curve at three points; their abscissa is the values of the initial angles of interest.

Accordingly, this trio angles are $\left\{32.51^{\circ}, 82.35^{\circ}, 87.12^{\circ}\right\}$. One needs to keep in mind that these angles are specific to the initial speed $37 \mathrm{~m} / \mathrm{s}$. In other words, these angles are speed-dependent.

The author would like to comment that although since Newton's era the projectile motion has been visited numerous times no such observation has been reported in scientific literature!

\section{Conclusion}

Scientific literature is flooded with information about the physics of the baseball. A thorough review of these resources reveals that there is no similarity between the outputs of the presented investigation vs. the historic body of knowledge. In this article we analyzed the impact of the drag force and the Magnus effect on the character of a projectile. Specifically we considered a baseball with a MLB character and included the impact of the speed-dependent drag coefficient. Depending on the choice of factors, four different scenarios were considered. Because of the complexity of the cases we heavily relied on the numeric solutions of the ODEs describing the motion. Results were interpreted and conclusions were reported for individual scenarios. For a comprehensive understanding, the results of each section are depicted graphically. In the last section we reported a new discovered result, namely a set of trio-angles conducive to an equal trajectory arc length. 
Table 2. The maximum trajectory arc lengths of a batted baseball at an initial speed of $37 \mathrm{~m} / \mathrm{s}$ vs. the corresponding initial angles for four scenarios of interest. The headings of the matrix explicitly describes the scenario of interest.

\begin{tabular}{|c|c|c|c|c|}
\hline & $\{\alpha D, \beta M\}=00$ & $\{\alpha D, \beta M\}=01$ & $\{\alpha D, \beta M\}=10$ & $\{\alpha D, \beta M\}=11$ \\
\hline “max length, $m-->”$ & 167.548 & 184.428 & 100.646 & 103.567 \\
\hline$\theta^{\circ}$ “-->” & $\theta \rightarrow 56.4556$ & $\theta \rightarrow 45.744$ & $\theta \rightarrow 62.166$ & $\theta \rightarrow 53.8664$ \\
\hline
\end{tabular}
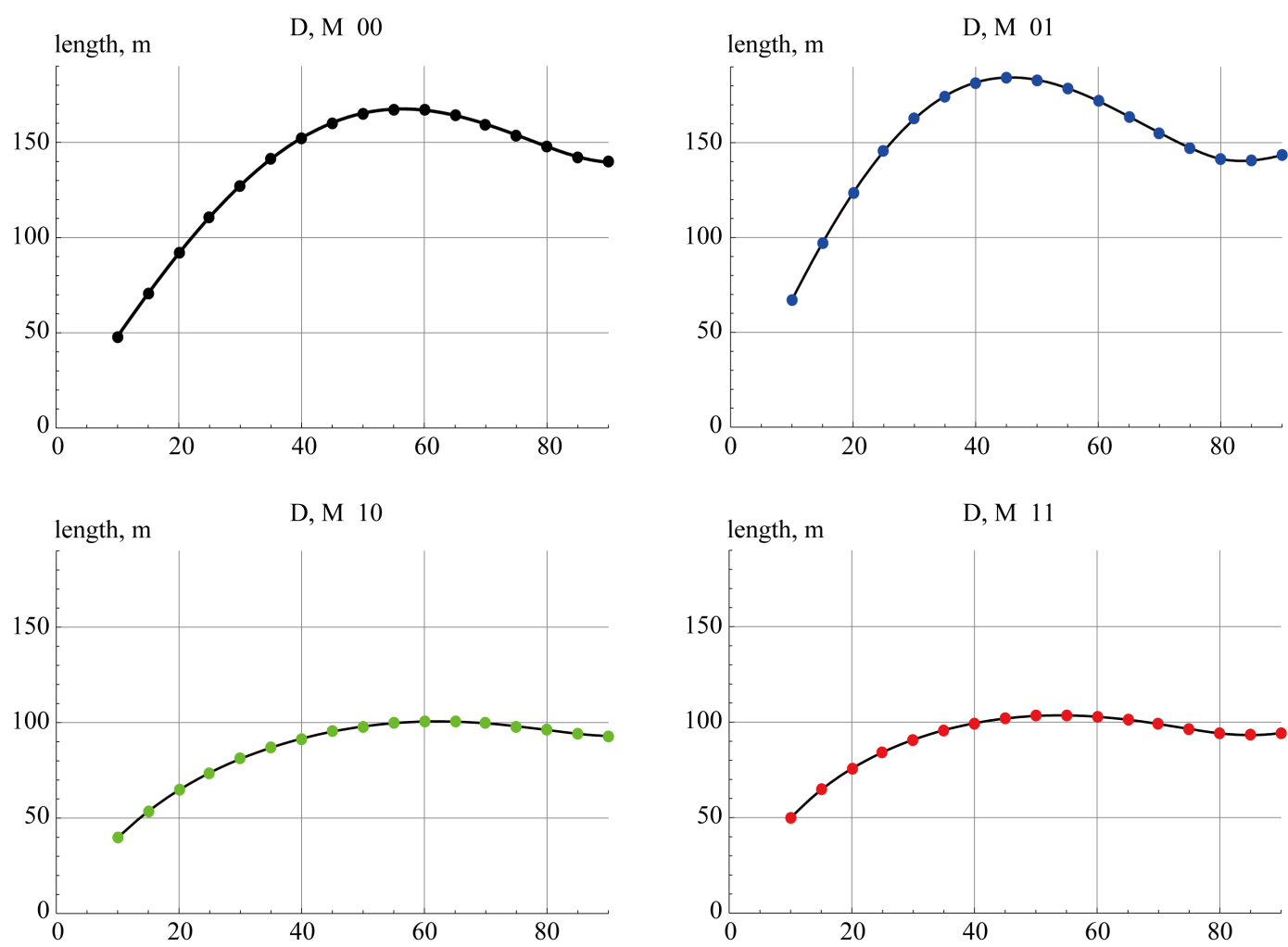

Figure 7. Plots of arc lengths of the trajectory of a baseball batted at $37 \mathrm{~m} / \mathrm{s}$ vs. the initial projected angle. The title of each plot describes the scenario of interest.

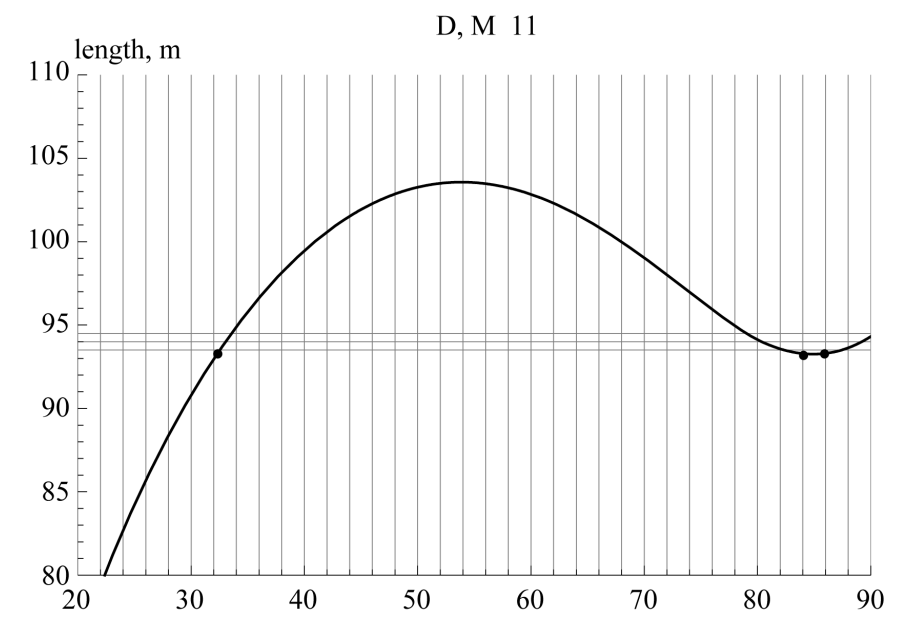

Figure 8. The abscissa of the three dots are the angles corresponding to the initial angles conducive to the same trajectory arc lengths. 


\section{Acknowledgements}

A version of this investigation was presented at the 11th International Conference on Computer Science and its Applications, Santander/Spain, 2011.

\section{References}

[1] Halliday, D., Risnick, R. and Walker, J. (2011) Fundamentals of Physics. 9th Edition, John Wiley \& Sons, Inc., Hoboken.

[2] Bauer, W. and Westfall, G. (2011) University Physics. McGraw-Hill, New York.

[3] Tipler, P. (1991) Physics. 3rd Edition, Worth Publisher, New York.

[4] VanWyk, S. (2008) Computer Solutions in Physics. World Scientific, Singapore.

[5] Giordano, N. and Nakanishi, H. (2006) Computational Physics. 2nd Edition, Prentice Hall, Upper Saddle River.

[6] Wolfgang, N. and Weidenmuller, H. (1980) Lecture Notes in Physics, Vol. 51. Springer-Verlag, Berlin, 194.

[7] Adair, R. (2002) The Physics of Baseball. 3rd Edition, Harper-Collins Publishers Inc., New York.

[8] Sarafian, H. (1999) On Projectile Motion. The Physics Teacher, 37, 86-88.

http://dx.doi.org/10.1119/1.880184 